\title{
Exquisite modulation of the active-site of Methanocaldococcus jannaschii adenylosuccinate synthetase in forward reaction complexes
}

\author{
Vishakha Karnawat $^{1}$, Sonali Mehrotra ${ }^{2}$, Hemalatha Balaram $^{2 *}$ and Mrinalini Puranik ${ }^{1 *}$ \\ ${ }^{1}$ Indian Institute of Science Education and Research, Pune-411008, India \\ ${ }^{2}$ Jawaharlal Nehru Centre for Advanced Scientific Research, Jakkur, Bangalore -560064, India \\ Manuscript submitted to: Biochemistry \\ Supplementary Information
}

*Corresponding authors:

Prof. Hemalatha Balaram, Jawaharlal Nehru Centre for Advanced Scientific Research, Jakkur, Bangalore -560064, India. Telephone: +91-80-22082812; Email: hb@jncasr.ac.in

Dr. Mrinalini Puranik, Chemistry, Indian Institute of Science Education and Research, Pune411008, Maharashtra, India. Phone: +91-735 069 4600; Email: mrinalini@iiserpune.ac.in; puranik.mrinalini@gmail.com 
Tables

Table S1: Comparison of shifts in wavenumber $\left(\mathrm{cm}^{-1}\right)$ observed in the UVRR spectra of IMP•MjADSS,

IMP•GTP•MjADSS, IMP•GTP•HDA•MjADSS and GTP•MjADSS complexes with respect to IMP in MES, pH 6.5.

\begin{tabular}{|c|c|c|c|c|c|c|c|c|c|}
\hline Assignments & IMP & $\begin{array}{l}\text { IMP• } \\
\text { MjADSS }\end{array}$ & Band & $\begin{array}{l}\text { IG or } \\
\text { IGH }^{\mathrm{a}}\end{array}$ & $\begin{array}{l}\text { IMP•GTP• } \\
\text { MjADSS }\end{array}$ & $\begin{array}{l}\text { IMP•GTP•HDA• } \\
\text { MjADSS }\end{array}$ & Assignments & GTP & $\begin{array}{l}\text { GTP• } \\
\text { MjADSS }\end{array}$ \\
\hline \multirow[t]{2}{*}{$\begin{array}{l}\text { Str } \mathrm{C} 6=\mathrm{O}(76 \%)+\mathrm{Be} \\
\mathrm{N} 1 \mathrm{H}+\mathrm{C} 6 \mathrm{~N} 1 \mathrm{C} 2(- \\
12 \%)\end{array}$} & 1690 & & $\begin{array}{l}\text { IMP + } \\
\text { GTP }\end{array}$ & 1687 & $1660(-27)$ & $1653(-34)$ & $\begin{array}{l}\text { Str } \mathrm{C} 6=\mathrm{O}+ \\
\mathrm{C} 5 \mathrm{C} 6+ \\
\text { BeN1H }\end{array}$ & 1684 & $\begin{array}{l}1657 \\
(-27)\end{array}$ \\
\hline & & & GTP & 1604 & $1616(12)$ & $1618(12)$ & $\begin{array}{l}\mathrm{Be} \mathrm{N} 1 \mathrm{H}+\mathrm{Str} \\
\mathrm{C} 2 \mathrm{~N}\end{array}$ & 1604 & $\begin{array}{l}1614 \\
(10)\end{array}$ \\
\hline \multirow[t]{2}{*}{$\begin{array}{l}\mathrm{Str} \mathrm{C} 2 \mathrm{~N} 3(55 \%)- \\
\mathrm{C} 4 \mathrm{C} 5-\mathrm{C} 5 \mathrm{C} 6+\mathrm{Be} \\
\mathrm{C} 2 \mathrm{H}(-12 \%)+\mathrm{N} 1 \mathrm{H}\end{array}$} & 1595 & $\begin{array}{l}1591 \\
(-3)\end{array}$ & IMP & 1595 & $1592(-3)$ & & & & \\
\hline & & & GTP & 1578 & $1573(-5)$ & $1578^{37}$ & $\begin{array}{l}\text { Str N3C4+ } \\
\text { C4C5 }+ \\
\text { C5N7 }\end{array}$ & 1578 & $\begin{array}{l}1574 \\
(-4)\end{array}$ \\
\hline $\begin{array}{l}\text { Str C4N9 }(12 \%)- \\
\text { N3C4 }(-22 \%)+\text { N7C8 } \\
+ \text { Be N9H }(15 \%)+ \\
\text { C8H }\end{array}$ & 1555 & $\begin{array}{l}1552 \\
(-5)\end{array}$ & IMP & 1555 & & & & & \\
\hline
\end{tabular}




\begin{tabular}{|c|c|c|c|c|c|c|c|c|c|}
\hline & & & GTP & 1537 & $1539(2)$ & $1536(-1)$ & $\begin{array}{l}\text { Str C4N9+ } \\
\mathrm{C} 6=\mathrm{O}+ \\
\mathrm{N} 1 \mathrm{C} 2\end{array}$ & 1537 & $\begin{array}{l}1534 \\
(-3)\end{array}$ \\
\hline & & & GTP & 1487 & $1481(-6)$ & $1481(-6)$ & $\begin{array}{l}\mathrm{Be} \text { C8H + } \\
\text { Str C8N9+ } \\
\text { N7C8 }\end{array}$ & 1487 & $\begin{array}{l}1483 \\
(-4)\end{array}$ \\
\hline \multirow[t]{2}{*}{$\begin{array}{l}\text { Be N1H }(17 \%)+C 2 H \\
(18 \%)+C 5 N 7 C 8(- \\
10 \%)+ \text { Str N1C2 } \\
(17 \%)-\text { C4N9 }(10 \%)\end{array}$} & 1421 & $\begin{array}{l}1418 \\
(-3)\end{array}$ & $\begin{array}{l}\mathrm{IMP}+\mathrm{GT} \\
\mathrm{P}\end{array}$ & 1417 & $1422(5)$ & $1418^{37}$ & $\begin{array}{l}\text { Str C4N9+ } \\
\text { C5N7 }\end{array}$ & 1413 & $\begin{array}{l}1417 \\
(+4)\end{array}$ \\
\hline & & & $\begin{array}{l}\mathrm{IMP}+\mathrm{GT} \\
\mathrm{P}\end{array}$ & 1364 & $1363(-1)$ & $1363(-1)$ & $\begin{array}{l}\text { Str N7C8+ } \\
\text { NIC6 }+ \\
\text { C5N7 }\end{array}$ & 1366 & $\begin{array}{l}1361 \\
(-5)\end{array}$ \\
\hline
\end{tabular}




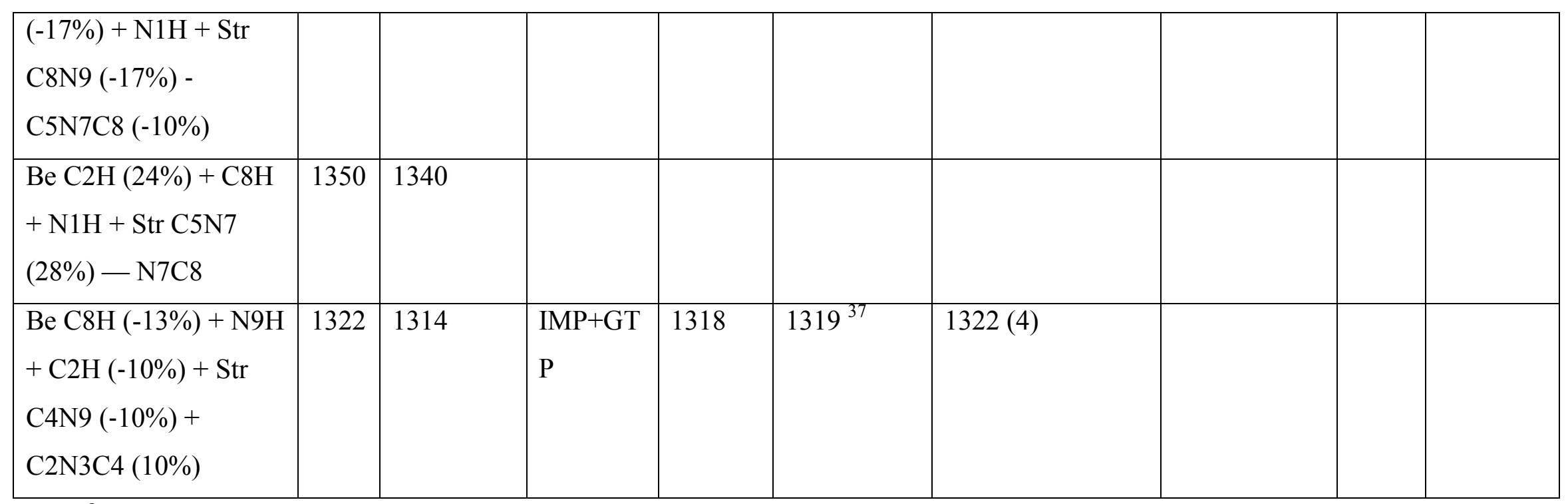

${ }^{\mathrm{a}}$ IG is (IMP+GTP) and IGH is (IMP+GTP+HDA). 


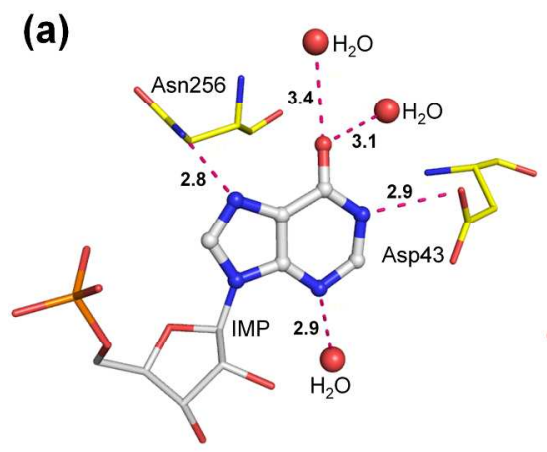

(b)

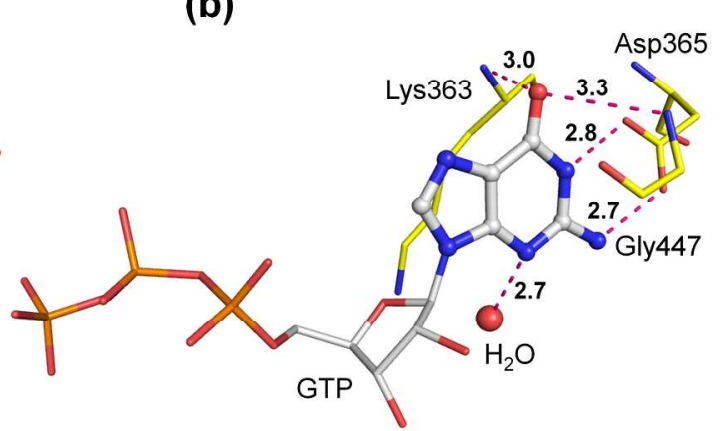

Figure S1: Non-covalent interactions at the active of mouse muscle ADSS. (a) IMP with activesite residue, taken from PDB: 1IWE: 2.10 $\AA$, (b) GTP with active-site residue taken from PDB: 1LOO: $2.20 \AA$. 


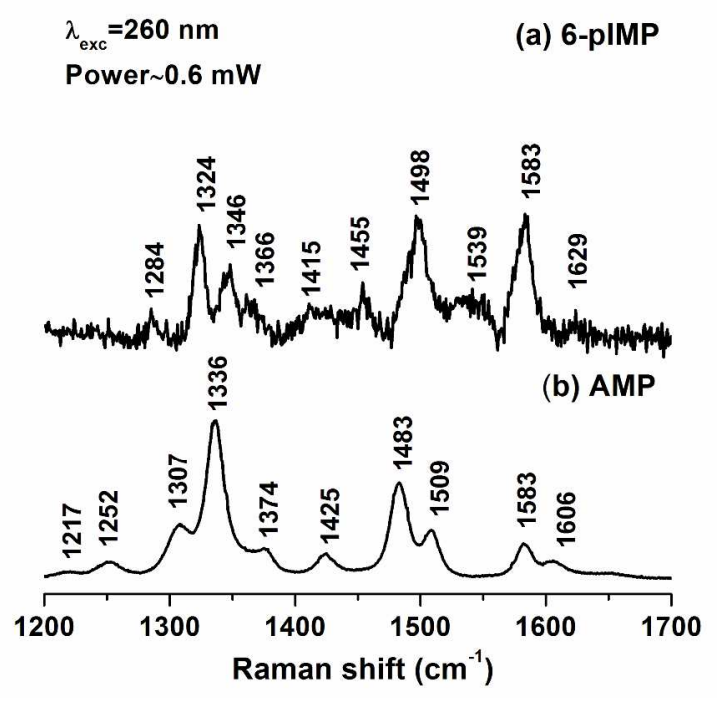

Figure S2: Resonance Raman spectra of 6-pIMP and its model compounds. (a) 6-pIMP obtained by subtracting the spectrum of GTP•MjADSS from that of IMP $\cdot G T P \cdot H D A \cdot M j A D S S$ spectrum and (b) AMP; acquired at the excitation wavelength of $260 \mathrm{~nm}$. 


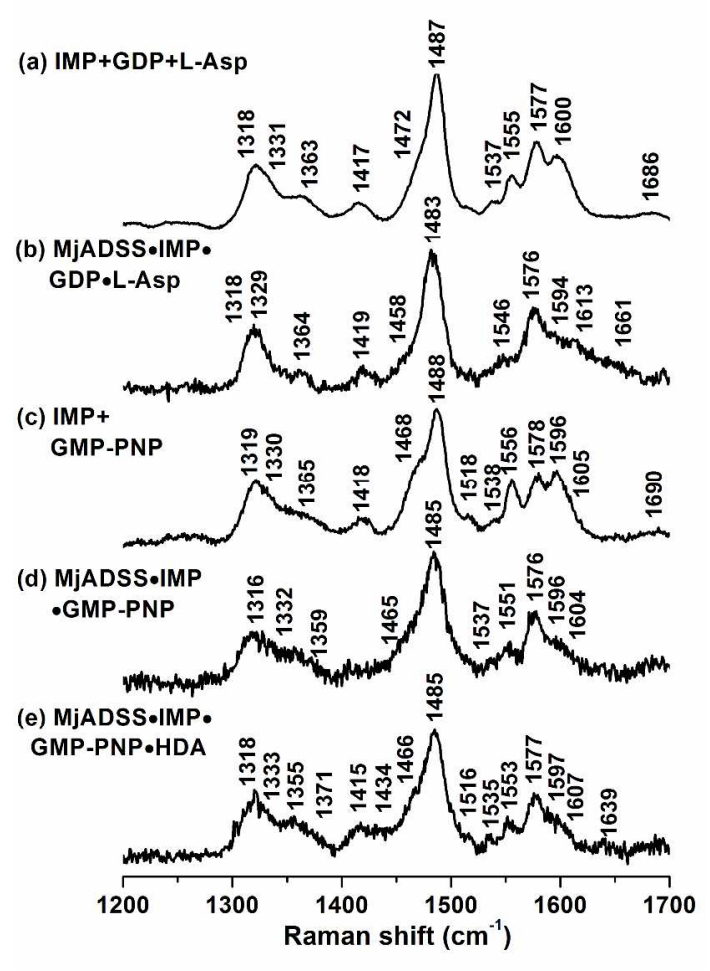

Figure S3: Resonance Raman spectra $\left(\lambda_{\text {exc }}=260 \mathrm{~nm}\right)$ of various guanine nucleotide complexes of MjADSS in MES buffer, $\mathrm{pH}$ 6.5. (a) (IMP+GDP+L-aspartate), (b) MjADSS $\cdot \mathrm{IMP} \cdot \mathrm{GDP} \cdot \mathrm{L}-$ aspartate, (c) (IMP+GMP-PNP), (d) MjADSS $\cdot \mathrm{IMP} \bullet \mathrm{GMP}-\mathrm{PNP}$, and (e) MjADSS•IMP•GMP$\mathrm{PNP} \cdot \mathrm{HDA}$. The characteristic band at $1499 \mathrm{~cm}^{-1}$ is not observed in any of these complexes. 